\title{
The Brief Case: Pneumonia Caused by Talaromyces marneffei
}

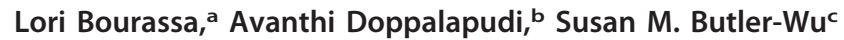 \\ aDepartment of Laboratory Medicine, University of Washington, Seattle, Washington, USA \\ bNorthside Hospital—Cherokee, Canton, Georgia, USA \\ cDepartment of Pathology and Laboratory Medicine, Keck School of Medicine of USC, Los Angeles, California, USA
}

KEYWORDS Penicillium marneffei, Talaromyces marneffei, penicilliosis, talaromycosis

\section{CASE}

A 45-year-old male was transferred to the emergency department (ED) following a syncopal event. He had struck his head on the ground but was found at the scene to be normotensive with normal blood sugar. Upon evaluation in the ED, the patient denied chest pain, palpitations, or shortness of breath. However, he reported a monthlong history of body aches and a mild cough. He had a history of human immunodeficiency virus (HIV) infection diagnosed in 2003 but was not on treatment until 2013, when he was diagnosed with Kaposi sarcoma of the skin and pneumocystis pneumonia. Antiretroviral therapy was subsequently initiated, but the patient had discontinued his medication 8 months prior. The patient denied any recent travel, but he had traveled to Northern Thailand approximately 18 months prior to presentation.

The patient reported a slight nonproductive cough but denied chest pain or shortness of breath, though his chest X-ray showed left lower lobe consolidation consistent with pneumonia. At the time of admission, his HIV load was 136,000 copies $/ \mathrm{ml}$, and he had a CD4 count of 9 cells $/ \mathrm{mm}^{3}$. Blood, urine, and stool cultures were negative for growth. Four sputum samples were obtained for bacterial culture but were rejected due to poor quality. Despite treatment with vancomycin, piperacillintazobactam, and levofloxacin, as well as prophylactic trimethoprim-sulfamethoxazole, fluconazole, and azithromycin, the patient continued to report subjective fevers. A chest computed tomography (CT) scan without contrast was obtained, and subtle opacities suggestive of bronchiolitis and a $2.5-\mathrm{cm}$ mass in the left lung were appreciated. The patient underwent bronchoscopy to determine whether the mass was due to an infection or neoplasia.

Cultures of the bronchoalveolar lavage fluid for bacteria and acid-fast bacilli were negative for growth. However, fungal cultures grew velvety yellow and tan-colored colonies on inhibitory mold agar (Hardy Diagnostics, Santa Maria, CA) after 3 days of incubation at $30^{\circ} \mathrm{C}$ (Fig. 1a). A diffusible deep red pigment was observed after 4 days of growth. A tape preparation of the colonies showed brush-like structures typical of the genus Penicillium, i.e., hyaline, septate hyphae with conidiophores with four to five short, broad metulae, each bearing four to six phialides with oval conidia (Fig. 1b). Because of the diffusible red pigment observed, the organism was subcultured to mycobiotic agar (Remel Thermo Scientific, Waltham, MA) and incubated at $37^{\circ} \mathrm{C}$. When incubated at $37^{\circ} \mathrm{C}$, the organism grew as white, dry, yeast-like colonies (Fig. 1c). Microscopic examination of the colonies revealed oval, yeast-like cells with a central septum (Fig. 1d). Based on the isolate's thermally dimorphic growth characteristics and diffusible red pigment, the isolate was presumptively identified as Talaromyces marneffei. Sequencing of the internal transcribed spacer (ITS) regions, ITS1 and ITS2, and the $28 \mathrm{~S}$ rRNA gene confirmed the identification.

The patient was treated with itraconazole ( $200 \mathrm{mg}$ twice a day) for 7 months, at which time a repeat chest $\mathrm{CT}$ revealed complete resolution of his left lower lobe

Citation Bourassa L, Doppalapudi A, Butler-Wu SM. 2019. The Brief Case: Pneumonia caused by Talaromyces marneffei. J Clin Microbiol 57:e01690-18. https://doi.org/10.1128/JCM .01690-18.

Editor Carey-Ann D. Burnham, Washington University School of Medicine Copyright $\odot 2019$ American Society for Microbiology. All Rights Reserved. Address correspondence to Lori Bourassa, bourassa@uw.edu.

For answers to the self-assessment questions and take-home points, see https://doi.org/10.1128/ JCM.01691-18 in this issue.

Published 26 April 2019 

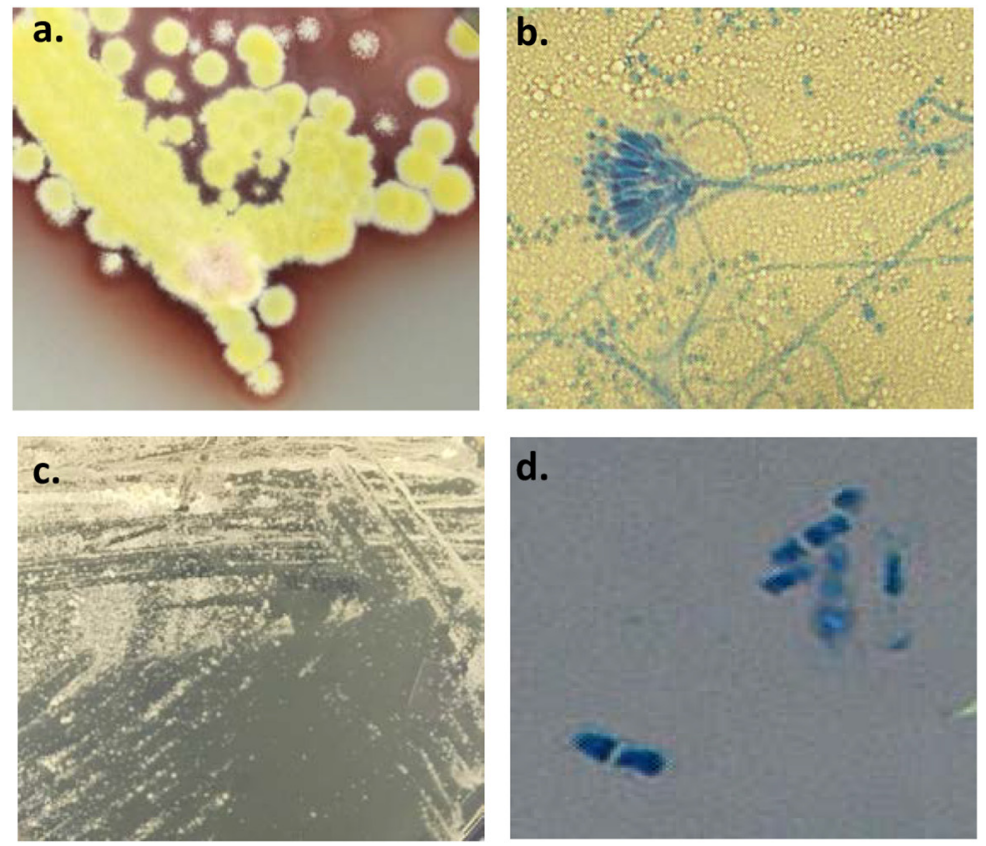

$37^{\circ} \mathrm{C}$

FIG 1 (a) Fungal cultures grew velvety yellow and tan-colored colonies on inhibitory mold agar (Hardy Diagnostics, Santa Maria, CA) after 3 days of incubation at $30^{\circ} \mathrm{C}$. (b) Tape preparation of the colonies showing brush-like structures typical of the genus Penicillium, i.e., hyaline, septate hyphae with conidiophores with four to five short, broad metulae, each bearing four to six phialides with oval conidia. (c) When incubated at $37^{\circ} \mathrm{C}$, the organism grew as white, dry, yeast-like colonies. (d) Microscopic examination of the colonies revealed oval, yeast-like cells with a central septum.

consolidation. The patient was transitioned to a maintenance dose of $200 \mathrm{mg}$ itraconazole daily until he had a CD4 count consistently $\geq 100$ cell $/ \mathrm{mm}^{3}$ for at least 6 months while on antiretroviral therapy. The patient was doing well at follow-up 1 year later.

\section{DISCUSSION}

Talaromyces marneffei (formally Penicillium marneffei) is a pathogenic, thermally dimorphic fungus that is a significant cause of morbidity and mortality among HIVinfected and other immunocompromised patients who reside in, or travel to, regions where this fungus is endemic (1-4). T. marneffei is endemic to southern China, Taiwan, Hong Kong, and other parts of southeast Asia, particularly Northern Thailand, Vietnam, Cambodia, and northeastern India (1, 2). It was first isolated from the hepatic lesions of the bamboo rat (Rhizomys sinensis) in Vietnam in 1956 (4). T. marneffei with genetic similarity to human T. marneffei isolates has since been found among bamboo rats in China, suggesting that bamboo rats are a reservoir of infection, although the definitive environmental reservoir is unknown $(1,2,5)$. The incidence of infection is known to peak during rainy seasons in areas of endemicity, with exposure to soil and decaying material thought to increase risk of infection $(1,2,6)$. However, attempts to culture $T$. marneffei from soil have been unsuccessful (1).

Human infection likely occurs by ingestion or inhalation of conidia into the lungs ( 1 , 5). Once in the lung, the conidia are phagocytosed by pulmonary alveolar macrophages and germinate into unicellular yeast cells $(1,2)$. Granulomas may be seen in the lung, liver, skin, and subcutaneous tissues of immunocompetent hosts, which may lead to a misdiagnosis of tuberculosis $(1,2,5)$. Once in the lung, $T$. marneffei can disseminate via a hematogenous route to other body sites, including the bone marrow and skin $(1,2)$. A case of laboratory acquired T. marneffei was also reported after accidental inoculation of the organism into a researcher's finger (2).

Prior to 2015, T. marneffei was known as Penicillium marneffei. The disease caused by T. marneffei was referred to as penicilliosis but is now called talaromycosis $(1,3)$. Talaromycosis is a systemic infection characterized by fungal invasion of multiple organ 
systems, including the lungs, blood, skin, and bone marrow and can be fatal if left untreated $(1,6)$. Talaromycosis is considered an AIDS-defining illness in areas of endemicity $(1,2,4)$. In some regions, $T$. marneffei ranks as the third most common opportunistic infection among HIV/AIDS patients alongside tuberculosis and cryptococcosis $(3,4)$. As HIV transmission has been reduced in areas of endemicity, a concomitant decrease in the incidence of talaromycosis has also been observed (2). In those with HIV/AIDS infection, T. marneffei infection typically develops when CD4 counts are less than 50 cells/ml (1). While talaromycosis is found mostly among HIV/AIDS patients, infections have also been reported in other immunocompromised patients, including individuals with systemic lupus erythematosus and solid organ and hematopoietic stem cell transplant recipients, as well as among hematology patients who received targeted therapy with monoclonal antibodies against CD20 or kinase inhibitors $(1,4)$. In some patients, symptoms may not develop until immunosuppression develops months to years after exposure to T. marneffei (4). Therefore, it is essential to collect a complete travel history to ensure there are no delays in diagnosis or treatment. While very rare, $T$. marneffei infection has been described in immunocompetent individuals (7).

The most common symptoms observed in patients with $T$. marneffei infection include fever, weight loss, malaise, and anemia $(5,6)$. Fungemia, lymphadenopathy, hepatomegaly, pulmonary disease (nonproductive cough and dyspnea), diarrhea, splenomegaly, and generalized skin lesions may also be present. Skin lesions commonly appear on the ears, face, extremities, and genitalia $(1,5,6)$. The prognosis is poor without treatment (100\% mortality), and while early treatment is essential to reduce mortality, mortality in treated patients ranges from 10 to $33 \%(1,3,4,6)$. The recommended treatments for talaromycosis are amphotericin B and itraconazole $(2,3)$. Although there are no antifungal susceptibility interpretive criteria for this organism, in vitro antifungal susceptibility testing may help to guide antifungal treatment (1). $T$. marneffei generally has low MIC values for most azoles, including voriconazole, itraconazole, and ketoconazole, as well as 5-flucytosine (1). However, T. marneffei tends to have elevated fluconazole MICs in vitro, and some strains are considered resistant (1). Clinical response to fluconazole treatment has been poor $(1,3,6)$.

Diagnosis of $T$. marneffei infection is made by visualization of the organism in clinical specimens by microscopy or by isolation of the organism from clinical specimens, including blood, skin lesions, lymph node biopsy specimens, bone marrow, sputum, bronchoalveolar lavage, urine, and stool $(1,2)$. Culture from bone marrow, skin biopsy specimens, and blood have demonstrated high sensitivity for the diagnosis of $T$. marneffei infection $(2,6)$. T. marneffei has been isolated from blood using automated blood culture systems with both standard or mycobacterial blood culture bottles, with an average time to positivity of 4 days (6). When cultured at $37^{\circ} \mathrm{C}, T$. marneffei grows as spherical to ellipsoidal yeast-like cells 2 to $6 \mu \mathrm{m}$ in diameter with a clearly defined central septum (1). The yeast-like cells reproduce by binary fission rather than budding, a characteristic that distinguishes $T$. marneffei from another thermally dimorphic fungus, Histoplasma capsulatum $(2,5)$. White to gray or tan yeast-like colonies appear after 2 to 4 days of growth $(1,7)$. At temperatures between 25 and $30^{\circ} \mathrm{C}, T$. marneffei grows as a filamentous fungus (i.e., mold). Microscopically, T. marneffei demonstrates the septate hyphae and conidiophores and conidia typical of Penicillium species $(1,6)$. Colonies appear rapidly and are often fluffy and white to tan. Yellowish-green conidial heads may be observed. Colonies tend to become brown or gray to green with age (1, 7). A diffusible red-brown pigment is evident in maturing colonies, particularly on the reverse (1). While the production of red pigment by ageing colonies is suggestive of $T$. marneffei, nonpathogenic Penicillium species, including P. citrinum, $P$. janthellum, $P$. purpurogenum, and $P$. rubrum, may also produce a similar red pigment (1). Conversion from the mold to yeast form with corresponding microscopic morphology is diagnostic of $T$. marneffei infection (1). T. marneffei shows temperature-dependent dimorphic growth and the only member of the genera Talaromyces that is a human pathogen ( 1 , 2 , 5). T. marneffei is a biohazard to laboratory staff (1). Slide cultures should not be 
performed; specimens and cultures should be handled in a biosafety level 3 containment or above $(1,7)$.

Although methods have been developed for the detection of T. marneffei-specific antibodies and antigens, no standardized commercial serological assays are available. Therefore, serology is not widely used for the diagnosis of T. marneffei infection $(1,2)$. Molecular diagnosis by PCR with sequencing or probe hybridization may be helpful in the identification of T. marneffei from clinical specimens (1).

In conclusion, talaromycosis should be considered in immunocompromised patients, particularly those with advanced HIV/AIDS, those who resided in or have traveled to regions where T. marneffei is endemic, and those who present with fever, weight loss, and anemia (1-4). Although T. marneffei morphologically resembles nonpathogenic Penicillium species, it exhibits thermally dimorphic growth and produces a diffusible red pigment while growing as a mold between 25 and $30^{\circ} \mathrm{C}(1,7)$. Diagnosis of $T$. marneffei infection can be made by visualization of the characteristic morphology of the spherical to ellipsoidal yeast-like cells in clinical specimens and demonstration of the conversion from mold to yeast forms and consistent microscopic morphology in cultured isolates (1).

\section{SELF-ASSESSMENT QUESTIONS}

1. T. marneffei infection occurs primarily in individuals who:

a. Are HIV-infected and reside in or have traveled to an area of endemicity

b. Are elderly

c. Are women of childbearing age

d. Have chronic lung disease

2. Which morphological form would be observed for suspected T. marneffei isolates when cultured at $37^{\circ} \mathrm{C}$ ?

a. Budding spherical to ellipsoidal yeast-like cells

b. A filamentous fungus with a diffusible red pigment

c. Spherical to ellipsoidal yeast-like cells with a central septum

d. Spherical to ellipsoidal yeast-like cells with a diffusible red pigment

3. Which antifungal drug would be the least effective for the treatment of talaromycosis?
a. Amphotericin B
b. Fluconazole
c. Voriconazole
d. 5-Flucytosine

\section{REFERENCES}

1. Chen SC-A, Sorrell TC, Wieland M. 2015. Aspergillus and Penicillium, p 2030-2056. In Jorgensen JH, Carroll KC, Funke G, Landry ML, Richter SS, Warnock DW (ed), Manual of clinical microbiology, vol 11. ASM Press, Washington, DC.

2. Vanittanakom N, Cooper CR, Jr, Fisher MC, Sirisanthana T. 2006. Penicillium marneffei infection and recent advances in the epidemiology and molecular biology aspects. Clin Microbiol Rev 19:95-110. https://doi.org/ 10.1128/CMR.19.1.95-110.2006.

3. Limper $A H$, Adenis A, Le T, Harrison TS. 2017. Fungal infections in HIV/ AIDS. Lancet Infect Dis 11:334-343.

4. Chan JF, Lau SK, Yuen KY, Woo PC. 2016. Talaromyces (Penicillium) marnef-

fei infection in non-HIV-infected patients. Emerg Microbes Infect 5:1-9. https://doi.org/10.1038/emi.2016.18.

5. Duong TA. 1996. Infection due to Penicillium marneffei, an emerging pathogen: review of 155 reported cases. Clin Infect Dis 23:125-130. https://doi.org/10.1093/clinids/23.1.125.

6. Wong SY, Wong KF. 2011. Penicillium marneffei infection in AIDS. Pathol Res Int 2011:764293. https://doi.org/10.4061/2011/764293.

7. Walsh T, Hayden RT, Larone DH. 2018. Talaromyces (Penicillium) marneffei, p 176-177. In Larone's medically important fungi: a guide to identification, 6th ed. ASM Press, Washington, DC. 